Supporting Information (SI)

\title{
Historical Black Carbon Reconstruction from the Lake Sediments of the Himalayan - Tibetan Plateau
}

Bigyan Neupane ${ }^{1,2}$, Shichang Kang ${ }^{1,2,3^{*}}$, Pengfei Chen ${ }^{1}$, Yulan Zhang ${ }^{1}$, Kirpa Ram ${ }^{4,5}$, Dipesh Rupakheti $^{1}$, Lekhendra Tripathee ${ }^{1}$, Chhatra Mani Sharma ${ }^{1,6}$, Zhiyuan Cong $^{3,4}$, Chaoliu Li ${ }^{3,4}$, Juzhi $\mathrm{Hou}^{3,4}$, Min $\mathrm{Xu}^{1}$, Poonam Thapa ${ }^{1,2}$

${ }^{1}$ State Key Laboratory of Cryospheric Science, Northwest Institute of Eco-Environment and Resources, Chinese Academy of Sciences, Lanzhou 730000, China

${ }^{2}$ University of Chinese Academy of Sciences, 100049, Beijing, China

${ }^{3}$ CAS Center for Excellence in Tibetan Plateau Earth Sciences, Beijing 100101, China

${ }^{4}$ Key Laboratory of Tibetan Environment Changes and Land Surface Processes, Institute of Tibetan Plateau Research, Chinese Academy of Sciences, Beijing 100101, China

${ }^{5}$ Institute of Environment and Sustainable Development, Banaras Hindu University, Varanasi221005, India

${ }^{6}$ Central Department of Environmental Science, Tribhuvan University, Kirtipur 44618, Nepal 


\section{*Corresponding author}

Prof. Dr. Shichang Kang

State Key Laboratory of Cryospheric Science, Northwest Institute of Eco-Environment and

Resources, Chinese Academy of Sciences, Lanzhou 730000, China.

Email: shichang.kang@lzb.ac.cn

Tel.: +86-0931-4967368 


\section{Description of Supporting Information:}

Number of pages: 12

Number of Figures: 5

Number of Tables: 2

Content included:

Page S4 Figure S1. Variability of excess ${ }^{210} \mathrm{~Pb}$ activity with depth for the sediment cores

Page S5 Figure S2. BC historical emission from 1850 to 2000

Page S6 Figure S3. Sedimentation rates of the investigated lakes and corresponding fluxes

Page S7 Figure S4. Annual average yearly precipitation $(\mathrm{mm})$ recorded from five closest meteorological stations from Lingge Co region obtained from 1962 to 2011

Page S8 Figure S5. Fire history map in the year $2003-2005$ during pre-monsoon season (March, April and May) in the region $\left(5^{\circ} \mathrm{N}-65^{\circ} \mathrm{E}, 55^{\circ} \mathrm{N}-135^{\circ} \mathrm{E}\right)$ acquired from MODIS Aqua and Terra satellites

Page S9 Table S1. Repeatability of the samples and BC values for Standard Reference Material (SRM), Marine Sediment, (NIST SRM-1941b) detected in this study

Page S10 Table S2. BC concentrations from each sediment core layer in all six lakes

Page S11 S1. BC Flux Determination

Page S12 References 

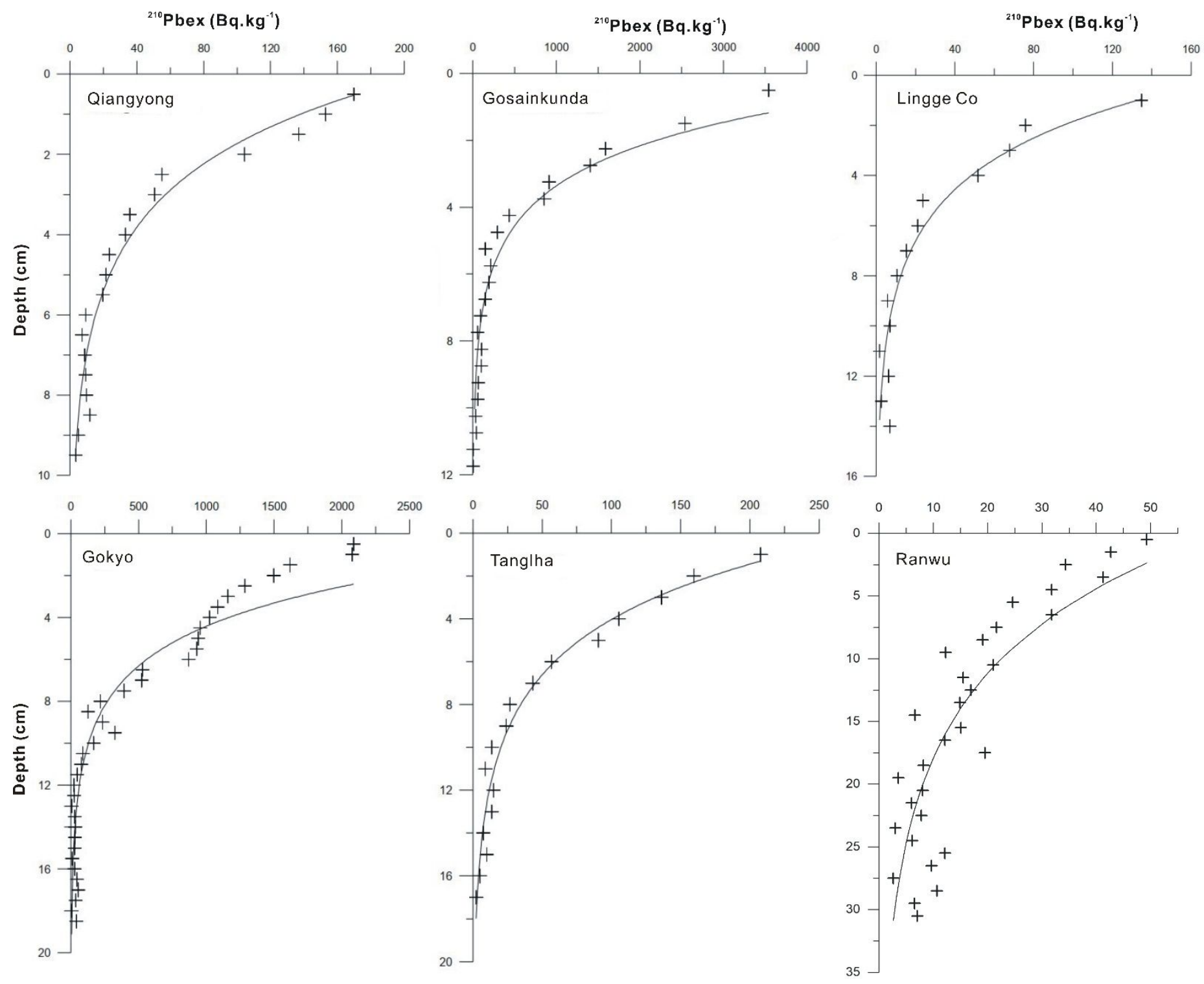

Figure S1. Variability of excess ${ }^{210} \mathrm{~Pb}$ activity with depth for the sediment cores ${ }^{1}$. Variability of excess ${ }^{210} \mathrm{~Pb}$ activity with depth in Lake Ranwu is based on this study. 


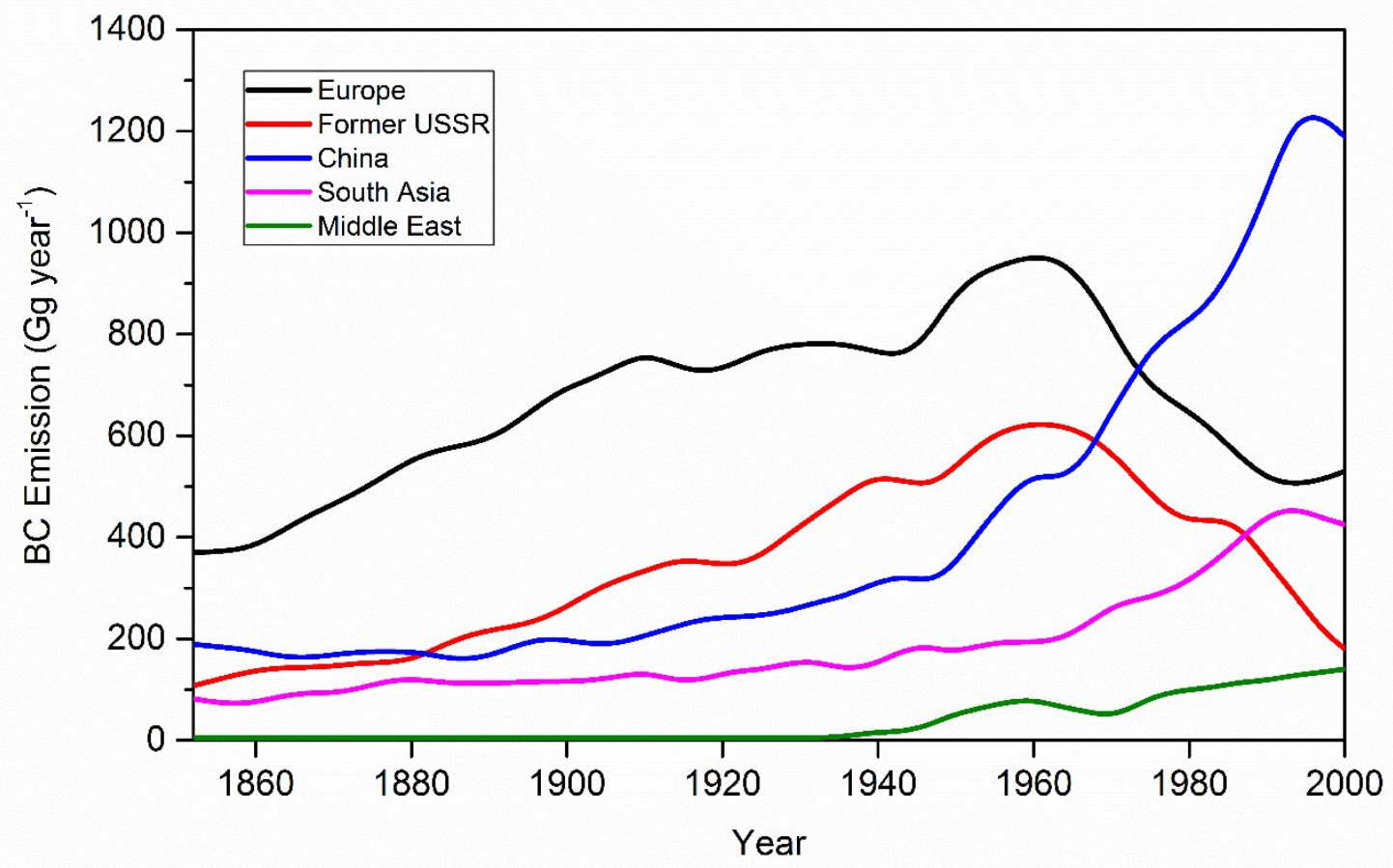

Figure S2. BC historical emission from 1850 to 2000 , redrawn ${ }^{2}$. 

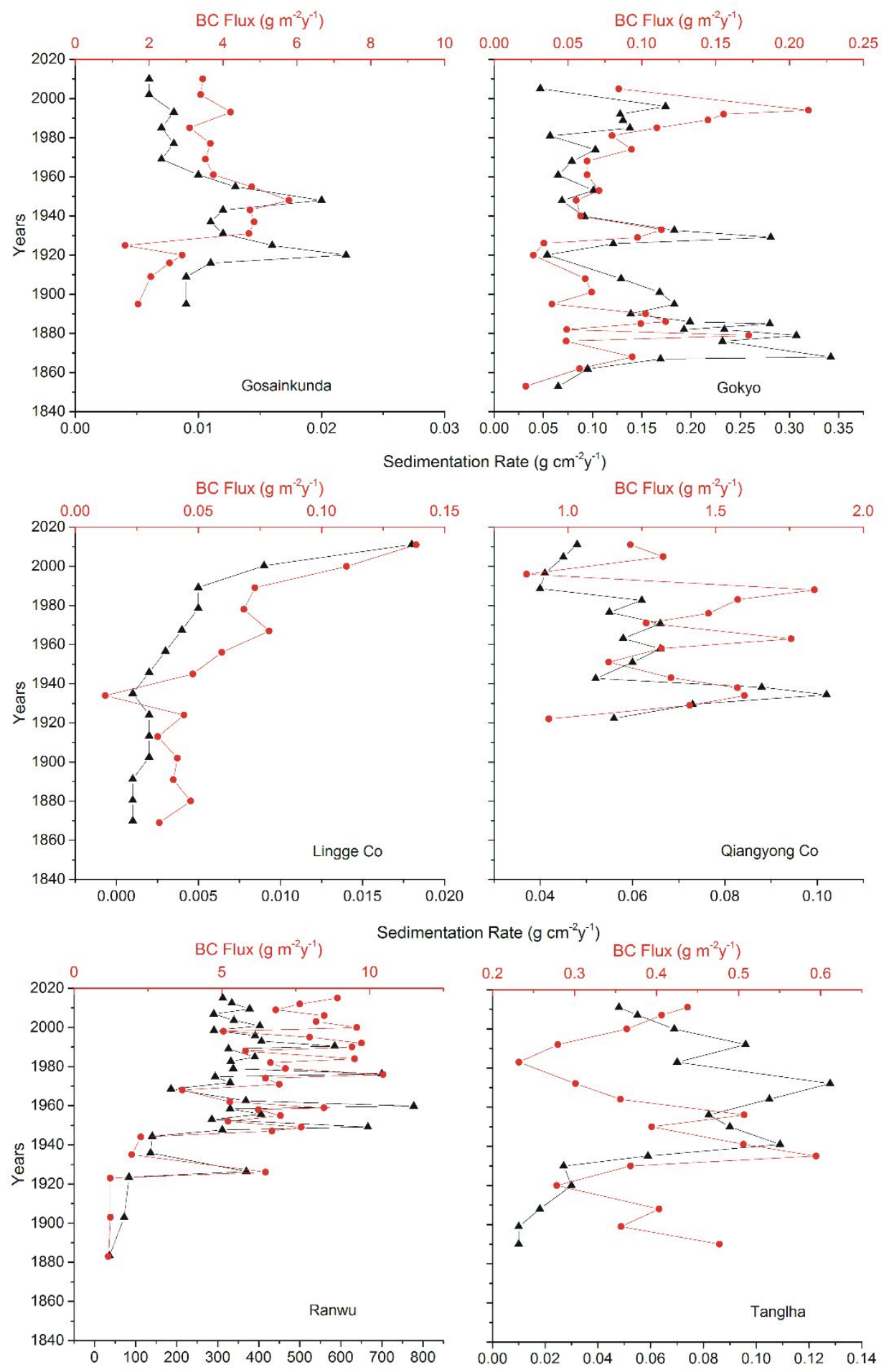

Sedimentation Rate $\left(\mathrm{g} \mathrm{cm}^{-2} \mathrm{y}^{-1}\right)$

Figure S3. Sedimentation rates of the investigated lakes and corresponding fluxes 

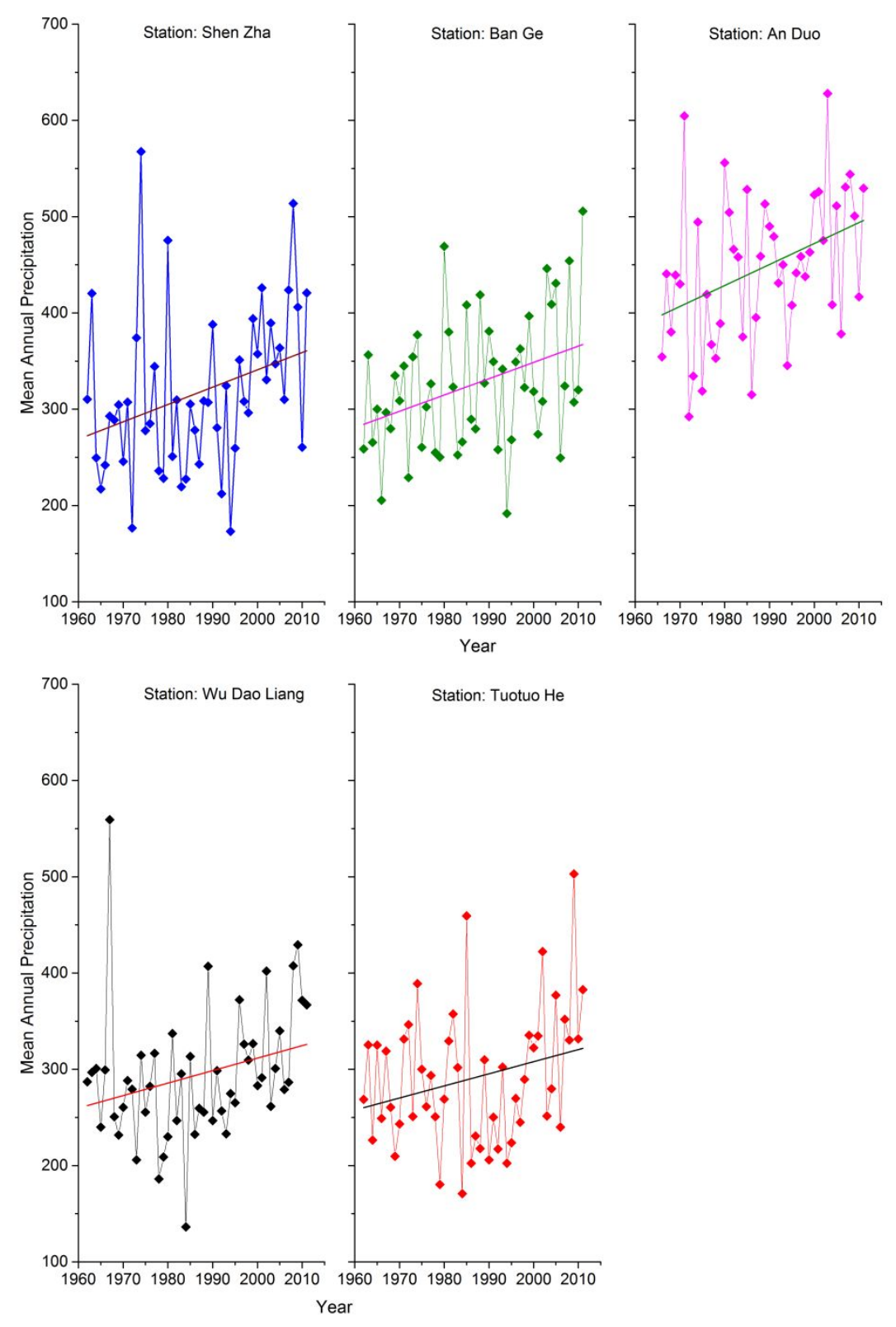

Figure S4. Annual average yearly precipitation $(\mathrm{mm})$ recorded from five closest meteorological stations from Lingge Co region obtained from 1962 to 2011. 

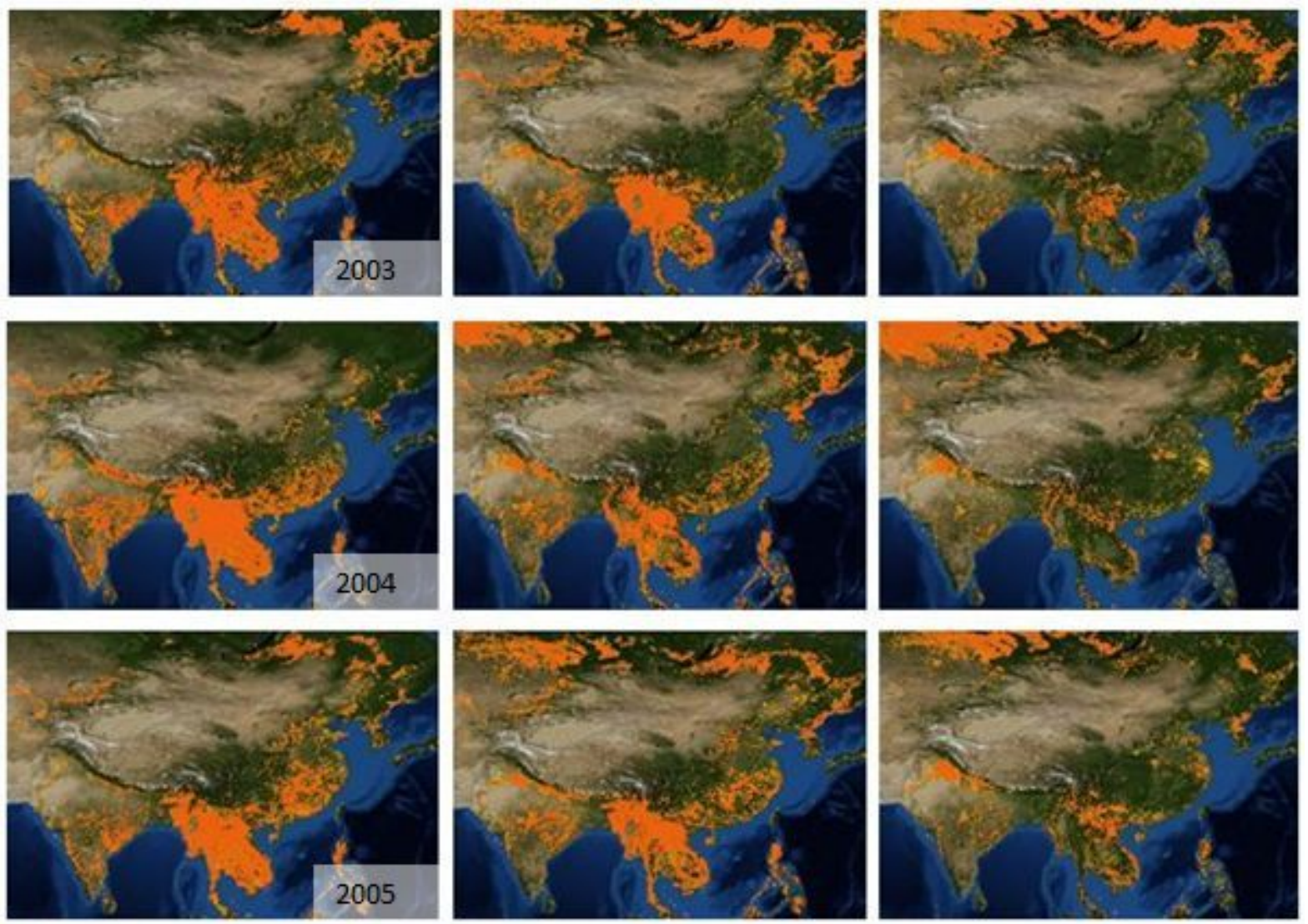

Figure S5. Fire history map in the year 2003 - 2005 during pre-monsoon season (March, April and May) in the region $\left(5^{\circ} \mathrm{N}-65^{\circ} \mathrm{E}, 55^{\circ} \mathrm{N}-135^{\circ} \mathrm{E}\right)$ acquired from MODIS Aqua and Terra satellites (https://firms.modaps.eosdis.nasa.gov/). 
Table S1. Reproducibility of the repeat samples $(\mathrm{n}=5)$ and $\mathrm{BC}$ values for Standard Reference Material (SRM), Marine Sediment, (NIST SRM-1941b) observed in this study.

\begin{tabular}{|c|c|c|c|c|c|c|c|}
\hline \multicolumn{4}{|c|}{ Reproducibility } & \multicolumn{4}{|c|}{ Accuracy } \\
\hline Sample ID & $\begin{array}{l}\mathrm{BC} \\
\left(\mathrm{mg} \mathrm{g}^{-1}\right)\end{array}$ & $\begin{array}{l}\text { Repeat } \\
\left(\mathrm{mg} \mathrm{g}^{-1}\right)\end{array}$ & $\%$ RPD* & $\begin{array}{l}\text { SRM } \\
\text { std }\end{array}$ & $\begin{array}{l}\mathrm{BC} \\
\left(\mathrm{mg} \mathrm{g}^{-1}\right)\end{array}$ & $\begin{array}{l}\text { Reported } \\
\left(\mathrm{mg} \mathrm{g}^{-1}\right)\end{array}$ & $\%$ RPD \\
\hline QY - 3 & 1.68 & 1.61 & 4.43 & SRM-1 & 13.1 & & 2.3 \\
\hline QY - 11 & 2.40 & 2.11 & 12.71 & SRM-2 & 12.7 & & 0.8 \\
\hline TGL - 4 & 0.83 & 0.80 & 4.46 & SRM-3 & 12.8 & & 0.0 \\
\hline RW - 1 & 1.53 & 1.76 & 13.76 & SRM-4 & 12.3 & & 4.0 \\
\hline \multirow[t]{4}{*}{ RW - 13} & 1.76 & 1.84 & 4.50 & SRM-5 & 11.7 & & 9.0 \\
\hline & & & & SRM-6 & 12.4 & & 3.2 \\
\hline & & & & SRM-7 & 12.1 & & 5.6 \\
\hline & & & & SRM-8 & 10.6 & & 18.8 \\
\hline Average & 1.64 & 1.62 & $7.97 \%$ & Average & 12.21 & 12.80 & $5.5 \%$ \\
\hline Std Dev & 0.50 & 0.45 & & Std Dev & 0.73 & 1.40 & \\
\hline \multicolumn{8}{|c|}{ *reported as average relative percentage deviation from the mean of two measurements } \\
\hline
\end{tabular}


Table S2. BC concentrations $\left(\mathrm{mg} \mathrm{g}^{-1}\right)$ from each sediment core layer in all six lakes

\begin{tabular}{|c|c|c|c|c|c|c|c|c|c|c|c|}
\hline \multicolumn{2}{|c|}{ Gokyo } & \multicolumn{2}{|c|}{ Gosainkunda } & \multicolumn{2}{|c|}{ Tanglha } & \multicolumn{2}{|c|}{ Qiangyong } & \multicolumn{2}{|c|}{ Lingge Co } & \multicolumn{2}{|c|}{ Ranwu } \\
\hline Year & BC & Year & BC & Year & BC & Year & BC & Year & BC & Year & BC \\
\hline 2005 & 0.307 & 2010 & 64.50 & 2011 & 1.269 & 2011 & 2.5261983 & 2011 & 0.639513 & 2015 & 1.765 \\
\hline 1994 & 0.245 & 2002 & 63.08 & 2007 & 1.012 & 2005 & 2.5797255 & 2000 & 0.503447 & 2012 & 1.717 \\
\hline 1992 & 0.240 & 1993 & 61.34 & 2000 & 1.079 & 1996 & 1.6862963 & 1989 & 0.4396 & 2009 & 1.639 \\
\hline 1989 & 0.226 & 1985 & 53.53 & 1992 & 0.841 & 1988 & 2.3627723 & 1978 & 0.287833 & 2006 & 2.004 \\
\hline 1985 & 0.224 & 1977 & 57.03 & 1983 & 0.817 & 1983 & 2.1747407 & 1967 & 0.34226 & 2003 & 2.048 \\
\hline 1981 & 0.306 & 1969 & 56.91 & 1972 & 0.818 & 1976 & 2.0071373 & 1956 & 0.250153 & 2000 & 1.583 \\
\hline 1974 & 0.267 & 1961 & 39.18 & 1964 & 0.935 & 1971 & 2.2009905 & 1945 & 0.2355 & 1998 & 1.405 \\
\hline 1968 & 0.183 & 1955 & 40.58 & 1956 & 1.068 & 1963 & 1.9595823 & 1934 & 0.152813 & 1995 & 1.702 \\
\hline 1961 & 0.211 & 1948 & 51.04 & 1950 & 0.981 & 1958 & 2.1277386 & 1924 & 0.183167 & 1992 & 1.469 \\
\hline 1953 & 0.203 & 1943 & 41.78 & 1941 & 0.826 & 1951 & 1.7825982 & 1913 & 0.146533 & 1990 & 1.624 \\
\hline 1948 & 0.155 & 1937 & 48.30 & 1935 & 0.946 & 1943 & 1.9979704 & 1902 & 0.17584 & 1988 & 1.379 \\
\hline 1940 & 0.162 & 1931 & 43.82 & 1930 & 1.009 & 1938 & 1.6107649 & 1891 & 0.186307 & 1984 & 1.534 \\
\hline 1933 & 0.165 & 1925 & 9.06 & 1920 & 0.985 & 1934 & 1.7546693 & 1880 & 0.20096 & 1982 & 1.756 \\
\hline 1929 & 0.100 & 1920 & 12.62 & 1908 & 1.031 & 1929 & 2.17288 & 1869 & 0.148627 & 1979 & 1.767 \\
\hline 1926 & 0.063 & 1916 & 26.73 & 1899 & 0.813 & 1922 & 1.5357455 & & & 1976 & 1.644 \\
\hline 1920 & 0.102 & 1909 & 29.66 & 1890 & 1.021 & & & & & 1974 & 1.643 \\
\hline 1908 & 0.178 & 1895 & 22.61 & & & & & & & 1971 & 1.480 \\
\hline 1901 & 0.109 & & & & & & & & & 1968 & 1.612 \\
\hline 1895 & 0.062 & & & & & & & & & 1962 & 1.170 \\
\hline 1890 & 0.132 & & & & & & & & & 1959 & 1.239 \\
\hline 1886 & 0.071 & & & & & & & & & 1958 & 1.542 \\
\hline 1885 & 0.057 & & & & & & & & & 1955 & 1.459 \\
\hline 1882 & 0.043 & & & & & & & & & 1952 & 1.478 \\
\hline 1879 & 0.194 & & & & & & & & & 1949 & 1.230 \\
\hline 1876 & 0.112 & & & & & & & & & 1947 & 1.490 \\
\hline 1868 & 0.139 & & & & & & & & & 1944 & 1.434 \\
\hline 1862 & 0.163 & & & & & & & & & 1935 & 1.154 \\
\hline \multirow[t]{4}{*}{1853} & 0.106 & & & & & & & & & 1926 & 1.343 \\
\hline & & & & & & & & & & 1923 & 1.418 \\
\hline & & & & & & & & & & 1903 & 1.627 \\
\hline & & & & & & & & & & 1883 & 1.309 \\
\hline
\end{tabular}




\section{S1. BC Flux Determination}

The method employed by Cong, et al. ${ }^{4}$ was used to determine flux in our study. The BC flux was calculated by multiplying the $\mathrm{BC}$ concentration (mg g-1) with the quotient of total weight of sample (dry weight per layer) and area of gravity corer $(6 \mathrm{~cm}$ inner diameter polycarbonate tube; area $=\pi \mathrm{r} 2$ ). The result was further divided by the deposition time (i.e. the difference between the time of deposition of the two consecutive layers) to obtain $\mathrm{BC}$ flux ( $\left.\mathrm{g} \mathrm{m}^{-2} \mathrm{yr}^{-1}\right)$.

BC Flux $=\frac{\text { BC concentration } \times \frac{\text { Dry wt. per layer }}{\text { Area of gravity corer }}}{\text { Deposition Time }}$ 


\section{References}

1. Kang, S.; Huang, J.; Wang, F.; Zhang, Q.; Zhang, Y.; Li, C.; Wang, L.; Chen, P.; Sharma, C. M.; Li, Q., Atmospheric Mercury Depositional Chronology Reconstructed from Lake Sediments and Ice Core in the Himalayas and Tibetan Plateau. Environmental science \& technology 2016, 50, (6), 2859-2869.

2. $\quad$ Bond, T. C.; Bhardwaj, E.; Dong, R.; Jogani, R.; Jung, S.; Roden, C.; Streets, D. G.; Trautmann, N. M., Historical emissions of black and organic carbon aerosol from energy-related combustion, 18502000. Global Biogeochemical Cycles 2007, 21, (2).

3. Han, Y.; Cao, J.; An, Z.; Chow, J. C.; Watson, J. G.; Jin, Z.; Fung, K.; Liu, S., Evaluation of the thermal/optical reflectance method for quantification of elemental carbon in sediments. Chemosphere 2007, 69, (4), 526-533.

4. Cong, Z.; Kang, S.; Gao, S.; Zhang, Y.; Li, Q.; Kawamura, K., Historical trends of atmospheric black carbon on Tibetan Plateau as reconstructed from a 150-year lake sediment record. Environmental science \& technology 2013, 47, (6), 2579-2586. 